\title{
$\alpha$-Linolenic Acid and Risk of Heart Failure: A Meta-Analysis
}

\author{
Jiandi Wu ${ }^{1 *}$, Min Qiu${ }^{2}$, Lichang Sun ${ }^{2}$, Jiangxiong Wen ${ }^{1}$, Dong-liang Liang ${ }^{1}$, Sulin Zheng ${ }^{2}$ \\ and Yuli Huang ${ }^{2 *}$
}

${ }^{1}$ Department of Cardiology, Affiliated Foshan Hospital, Southern Medical University, Foshan, China, ${ }^{2}$ Department of Cardiology, Shunde Hospital, Southern Medical University, Foshan, China

\section{OPEN ACCESS}

Edited by:

Chen Liu,

The First Affiliated Hospital of Sun Yat-sen University, China

Reviewed by:

Jun Lyu,

First Affiliated Hospital of Jinan

University, China

Kelsi Anderson,

Baylor College of Medicine,

United States

*Correspondence:

Jiandi Wu

fsdrwu@126.com

Yuli Huang

hyuli821@smu.edu.cn

Specialty section

This article was submitted to General Cardiovascular Medicine, a section of the journal

Frontiers in Cardiovascular Medicine

Received: 02 October 2021

Accepted: 22 November 2021

Published: 04 January 2022

Citation:

Wu J, Qiu M, Sun L, Wen J, Liang D-I,

Zheng $S$ and Huang $Y$ (2022) $\alpha$-Linolenic Acid and Risk of Heart

Failure: A Meta-Analysis.

Front. Cardiovasc. Med. 8:788452.

doi: 10.3389/fcvm.2021.788452
Background: The $\alpha$-linolenic acid is a plant origin $n-3$ fatty acid that may reduce the risk of cardiovascular disease. However, the effect of $\alpha$-linolenic acid (ALA) on the risk of heart failure $(\mathrm{HF})$ remains unclear. In this meta-analysis, we aimed to determine the role of ALA in the risk of incident HF.

Methods: Electronic databases were searched for studies up to August 10, 2021. Studies were included for meta-analysis if the adjusted risk of HF in different dietary intake or circulating levels of ALA was reported. We used the random-effects model to calculate the estimated hazard ratios (HRs) and 95\% Cl for higher ALA.

Results: A total of 6 studies (7 cohorts) comprising 135,270 participants were included for meta-analysis. After a median follow-up duration of 10 years, 5,905 cases of HF were recorded. No significant heterogeneity was observed among all the included studies. Random-effects model analyses showed that there was no significant association between ALA and the risk of incident HF, either assessed as quintiles (highest quintile vs. lowest quintile: $\mathrm{HR}=0.95,95 \% \mathrm{Cl}=0.86-1.06)$ or per $1 S D$ increment $(H R=0.99$, $95 \% \mathrm{Cl}=0.95-1.01)$. Furthermore, we did not observe any association between ALA and the risk of $\mathrm{HF}$ in subgroup analyses performed according to age, sex, follow-up duration, and measuring method of ALA.

Conclusions: We found no association between ALA and the risk of incident HF, suggesting that ALA might not be effective in the prevention of HF.

Keywords: $\alpha$-linolenic acid, fatty acid, heart failure, risk, meta-analysis

\section{INTRODUCTION}

Heart failure has become a growing global public health burden, with high rates of re-hospitalisation and mortality. Heart failure (HF) incidence increases dramatically with a growing ageing population, it was estimated that nowadays, there were more than 37.7 million HF patients worldwide (1). Although there have been significant improvements in the management of HF, the morbidity and mortality in HF patients were still high, which underscores the importance of the identification of novel risk factors and treatment modalities of HF (2-4).

Long-chain (LC) omega-3 polyunsaturated fatty acids (n-3 PUFA), including eicosapentaenoic acid (EPA) and docosahexaenoic acid (DHA), may play a protective role in the incidence and progression of HF, (5-7) through multiple mechanisms including anti-inflammation effects, improving cardiomyocytes' energy metabolism endothelial function (8). 
However, considering the sustainable supply and safety of the marine sources, plant-based n-3 PUFA is gaining much attention recently and is considered as an important alternative to n-3 PUFA sources (9). $\alpha$-linolenic acid (ALA), a plant origin essential n-3 fatty acid, can be converted to EPA and DHA. Therefore, it is important to explore whether ALA has similar effects on the risk of HF. However, although previous studies showed that dietary patterns that included ALA-rich foods may reduce the risk of cardiovascular disease $(10,11)$, the association between ALA and HF was unclear. The Physicians' Health Study has found that dietary ALA intake level showed an inverse trend towards an association with HF, (12) while other studies did not find a similar association (13-15). To address the knowledge gap, we performed a pooled analysis of observational studies to generate data on the associations between ALA and the risk of incident HF.

\section{METHODS}

\section{Databases Search Strategy and Inclusion Criteria of Studies}

We performed the meta-analysis following the recommendations of the Meta-analysis of Observational Studies in Epidemiology (MOOSE) group (16). Electronic databases, including PubMed, Google scholar, Cochran Library, and EMBASE, were searched for studies from inception until 31 July 2021, using a combined text and medical subheadings (MeSH) search strategies, with multiple terms related to "Omega-3 PUFA" or " $\alpha$-linolenic acid" and "heart failure". We were restricted to human studies. Reference lists of the related articles and included studies were manually reviewed to identify potentially missed pieces of research. The detailed strategy used for searching PubMed is presented in Supplementary File 1. When searching other databases, the strategies were similar.

We included observational studies for meta-analysis if they met the following criteria: (1) observational studies (including cohort studies, nest case-control studies, and post hoc analyses of randomised controlled trials) with adult participants (aged $\geq 18$ years); (2) dietary intakes or circulating levels of ALA were detected; (3) the risk of HF associated with different levels of ALA were reported.

We excluded studies if: (1) they were cross-sectional studies; (2) the risk of HF was unadjusted for confounders; (3) the followup duration was $<1$ year; (4) duplicated articles were reported from the same cohort dataset. If more than one report used data from the same participants' cohort, only the latest published article was included.

\section{Data Extraction and Quality Assessment of the Included Studies}

Two investigators (J.W. and M.Q.) independently conducted article searches and screened the retrieved articles. Full copies of potentially suitable studies were reviewed. Key information of the included studies, such as country, cohort characteristics, methods for measurement of ALA, study sample, sex proportion, mean/median age, HF cases, and follow-up duration was recorded. If the essential message was not reported, we contacted the principal authors for clarification.

The Newcastle-Ottawa Quality Assessment Scale for observational cohort studies was used to determine the study quality (17), which is based on: the selection (3 items with 1 point each), comparability (1 item with up to 2 points), and exposure/outcome (3 items with 1 point each), with the total score from 0 to 9 . Included studies were classified as good quality ( $\geq 7$ points), fair quality ( $4-6$ points), or poor ( $<4$ points), respectively $(18,19)$.

\section{Data Synthesis}

The outcome evaluated in this analysis was the risk of incident HF associated with different levels of dietary intake or circulating ALA. We extracted outcome data which adjusted for the maximal number of potential confounders for analysis. In the included studies, the associations of the HF risk and ALA levels were reported in different ways, such as per quartiles or quintiles of ALA levels, or per $1 S D$ increment in the continuous trait. Therefore, we used two methods to transfer the data into consistent comparisons. We compared the hazard ratio (HR) of HF risk in participants with the highest quintile of ALA with those with the lowest quintile and calculated the HR as per $1 S D$ increment in ALA. If effect measures were not reported as per quintile or per $1 S D$ change in the included studies, we converted the data following the previously published methods (20). Briefly, we assumed that the exposure variable (ALA) was with normal distribution and its association with the interesting outcome (risk of HF) was log-linear. The expected difference in means of the highest vs. lowest tertile is $2.18 \mathrm{SDs}$, and $2.54 \mathrm{SDs}$ for the highest vs. lowest quartile, $2.8 \mathrm{SDs}$ for the highest vs. lowest quintile, respectively (20). Therefore, HR reported as top vs. bottom quintile comparison can be converted to per $1 S D$ change by using a division conversion factor of 2.8 to the log HR. Accordingly, HRs for comparisons of extreme tertiles and quartiles are divided by 2.18 and 2.54, respectively. Conversely, HRs and 95\% CIs reported as comparisons of quartiles or tertiles can be converted to comparisons of quintiles, by using a multiplication conversion factor of $2.8 / 2.54$ or $2.8 / 2.18$ to the log HRs.

The inverse variance approach was used to calculate the log HRs and corresponding SEs with the random-effects models (21). The $\mathrm{I}^{2}$ statistics were used to test heterogeneity. An $\mathrm{I}^{2}$ value $>$ $50 \%$ was regarded as with significant heterogeneity among the included studies (2). Subgroup analyses of the primary outcome were conducted according to sex, age, follow-up period, and measure of ALA. We evaluated the publication bias by inspecting the funnel plots, as well as by using Egger's test and Begg's test. Finally, we performed the sensitivity analyses by changing the random-effects model into the fixed-effects model in the pooled analysis. We also recalculated the HRs by excluding one study at a time to determine the effect of every study on the pooled risk.

All analyses were performed using Stata 15.0 (StataCorp LP, College Station, TX, USA) and RevMan 5.3 (The Cochrane Collaboration, Copenhagen, Denmark). All $P$-values are twotailed, and a $P<0.05$ is considered as with statistical significance. 


\section{Potentially relevant articles identified and screened for retrieval $(n=1263)$}

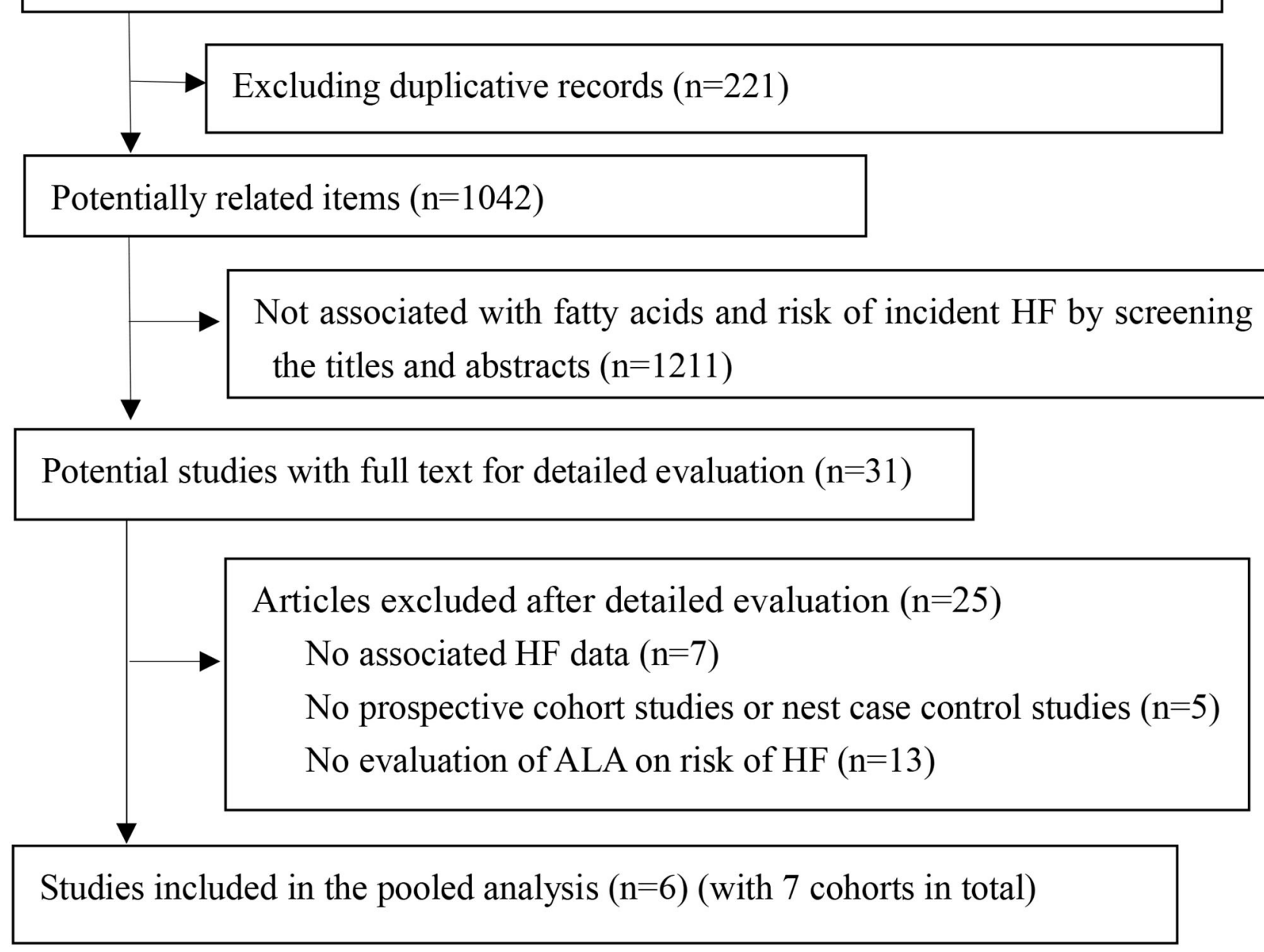

FIGURE 1 | The flow of papers through review. ALA, $\alpha$-Linolenic acid; HF, heart failure.

\section{RESULTS}

\section{Studies Characteristics and Quality}

We retrieved 1,263 article items from the searched electronic databases. After removing duplication records, two authors independently screened the titles and abstracts. Thirty-one articles were fully evaluated, and 6 studies were included in the pooled analysis finally (Figure 1 ). In the included studies, two of them reported dietary intake of ALA $(14,22), 3$ reported circulating ALA level $(12,15,23)$, and only one study reported both dietary and circulating ALA level (13). The 6 studies (7 cohorts) included 135,270 participants for analysis, with their main characteristics resented in Table 1. One study only enrolled men, two studies only enrolled women, while all other studies enrolled both genders. After the median follow-up duration of 10 years (ranged from 8.4 to 14.3 years), 5,905 cases of HF were recorded. The adjusted confounders for HF risk in the pooled studies are presented in Supplementary File 5. Only one study was graded as fair, and all other 5 studies were considered as of good quality according to our predefined quality assessment criteria (Supplementary File 3).

\section{ALA and Risk of Incident HF}

No statistical heterogeneity observed among all the included studies for the association between ALA and incident HF $\left(\mathrm{I}^{2}=0 \%, \quad P=0.77\right)$. Random-effects model analyses showed that there was no significant association between ALA and risk of incident HF, either assessed as quintiles (Figure 2, highest quintile vs. lowest quintile: $\mathrm{HR}=0.95$, 95\% CI $=0.86-1.06, P=0.36)$ or per $1 S D$ increment (Figure 3, HR $=0.99,95 \% \mathrm{CI}=0.95-1.01, P=0.36$ ). We did not find any evidence of publication bias by the funnel plot (Supplementary Files 4, 5), Egger's or Begg's tests (both $P>0.1)$. 
TABLE 1 | Characteristics of studies on ALA and risk of HF.

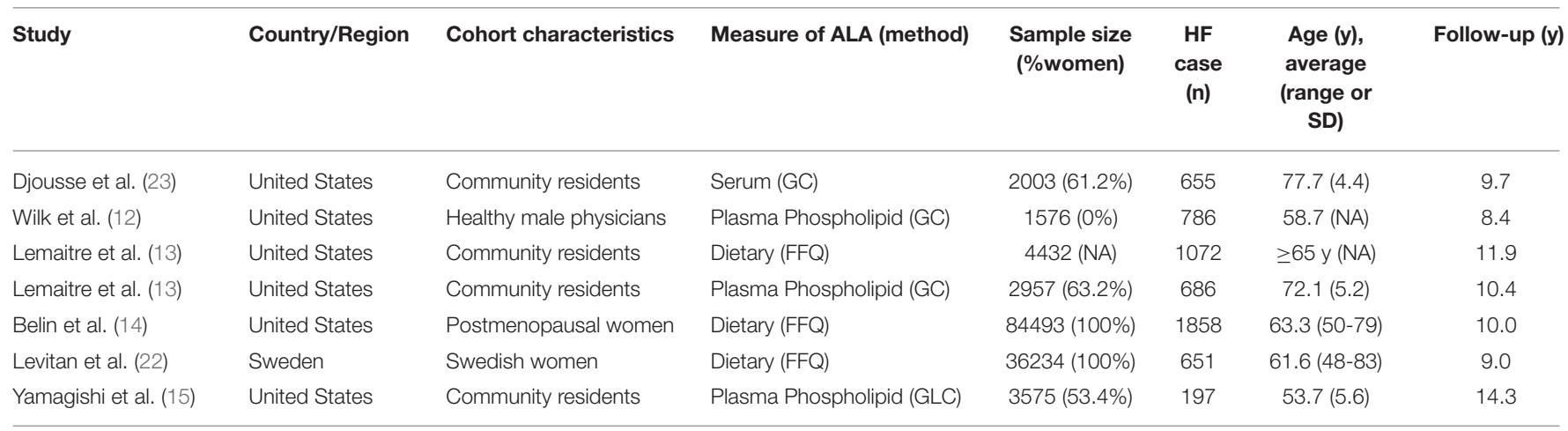

ALA, a-linolenic acid; ARIC, Atherosclerosis Risk in Communities; FFQ, food frequency questionnaire; GC, gas chromatography; GLC, gas-liquid chromatogram; HF, heart failure; NA, not available.

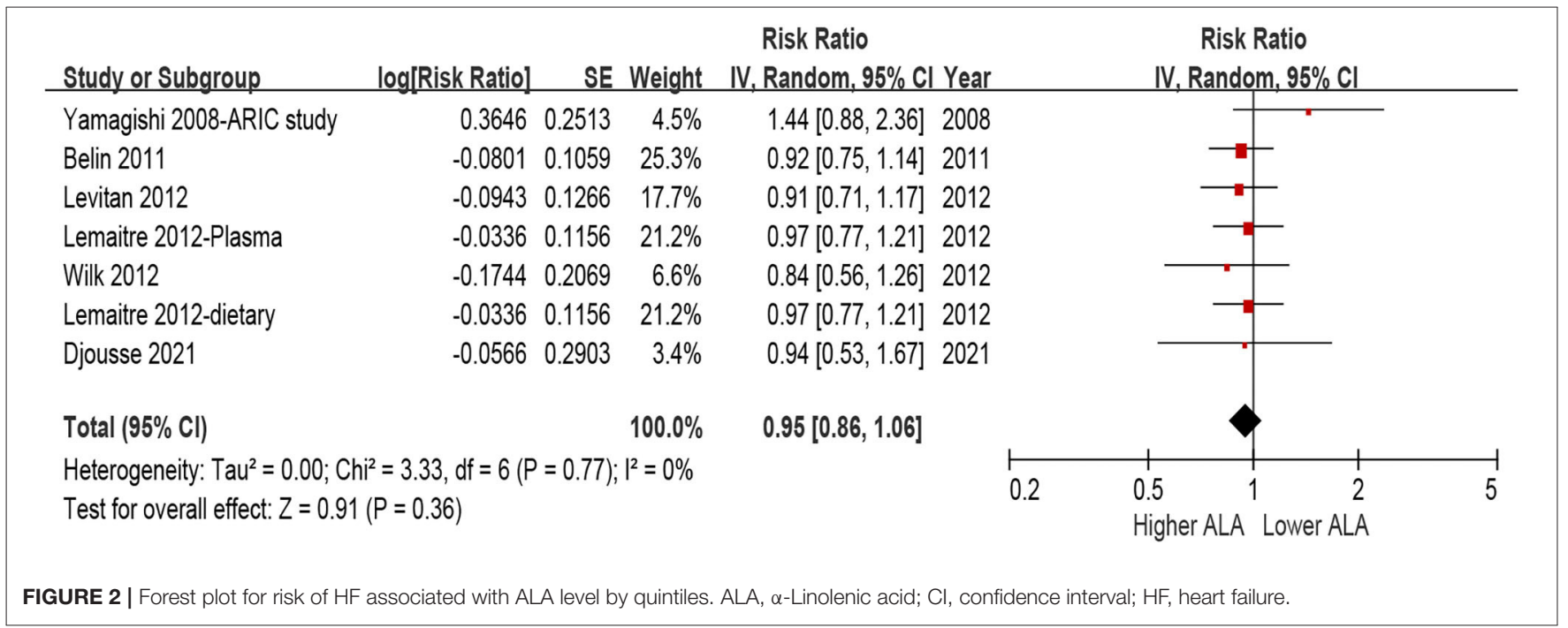

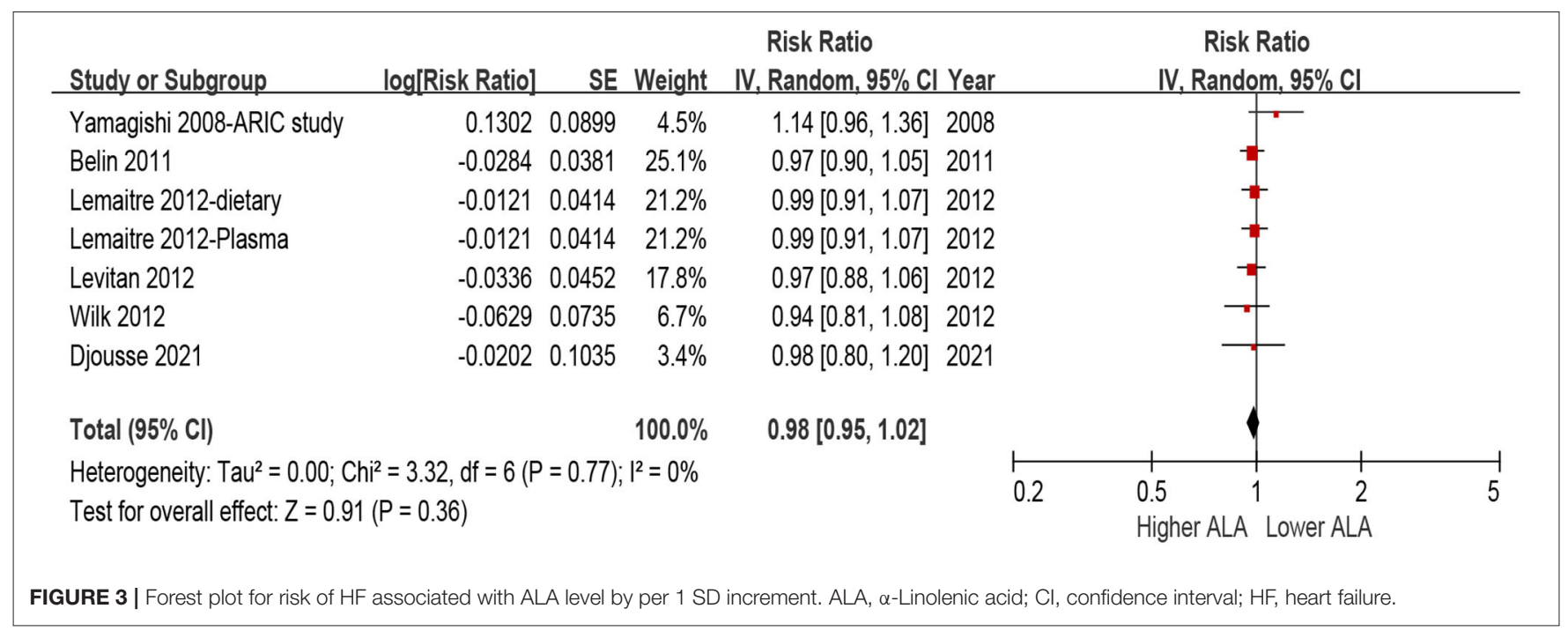




\section{Sensitivity Analyses and Subgroup Analyses}

We conducted several sensitivity analyses to confirm the HF outcome was consistent by using the fixed-effects models instead of random-effects models for meta-analysis or recalculating the estimated HRs and 95\% CI by excluding one study at each time. Furthermore, in subgroups performed according to age, sex, follow-up duration, and measuring method of ALA, we did not observe any significant association between ALA levels and the risk of incident HF. Furthermore, no significant heterogeneity was observed among all subgroup comparisons (all $\mathrm{I}^{2}=0 \%, P \geq 0.48$, Table 2).

\section{DISCUSSION}

To the best of our knowledge, this is the first pooled analysis evaluating the association between ALA and the risk of incident HF. We did not find any association between ALA (neither detected by dietary intake nor circulating level) and the risk of HF. The null result was further supported by no heterogeneity observed among multiple subgroup comparisons.

Basic research on ALA on cardiac function had yielded inconsistent results. In a mice/rat model with doxorubicinmediated cardiotoxicity, the prophylactic administration of the ALA was cardioprotective by decreasing inflammation, apoptosis, and mitochondrial dysfunction $(24,25)$. Enrichment of ALA in rodent diet reduced oxidative stress and inflammation, resulted in less left ventricle dilation, and decreased myocardial fibrosis during myocardial infarction $(26,27)$. In diabetic or obese mice, dietary ALA counters cardioprotective dysfunction mainly through anti-inflammatory effects $(28,29)$. ALA may also confer beneficial anti-platelet and anti-inflammatory effects, and interaction with gut microbiota, which is significantly associated with the risk HF $(20,30)$. However, these effects were not observed in a rat model under pressure overload conditions (31).
In humans, evidence suggests that increasing ALA is associated with favourable cardiometabolic status and lower inflammation status $(32,33)$, which may play a protective role in cardiac function. Data from NHANES found that ALA and DHA can enhance the negative association between maximal oxygen uptake and $\mathrm{C}$-reactive protein, suggesting that the antiinflammatory response to maximal oxygen uptake capacity is related to levels of ALA and (34). However, in a randomised clinical trial, it was also found that increasing plasmatic ALA had no impact on plasma inflammatory biomarkers (35). Taking these studies with our results together indicates that humans may not benefit from ALA intake in the view of prevention of HF.

In contrast, marine n-3 PUFA had been shown to be protective for $\operatorname{HF}(12,13,36)$. In humans, ALA can be bioconverted into EPA and DHA, however, the rate is not sufficiently. The rate of bioconversion from ALA to DHA is about $0.05-0.5 \%$, and to EPA is $0.2-10 \%$ (9). The limited bioconversion rate may limit the potential cardioprotective effects. Furthermore, this conversion rate from ALA to EPA/DHA was influenced by sex and genetic factors (especially for variants of desaturase and elongase enzymes), as well as dietary intake level of EPA/DHA. In a Danish cohort with 53,909 participants followed for a median of 13.4 years, an inverse association between dietary ALA intake and the risk of CVD was observed in individuals with a low intake of marine n-3 PUFA, while not in those with a higher intake of marine n-3 PUFA. These findings suggested that ALA may be associated with a lower risk of CVD only in those individuals with not adequate intake of marine n-3 PUFA (37). However, whether the relationship between ALA and the risk of incident HF was similar remains unexplored.

Our study has several major strengths. First, most of the included studies were adjusted for multiple confounders, including traditional cardiovascular risk factors and energy intake. This may mitigate the effect of potential confounders influencing the results. Second, the associations were consistently determined by category (highest quintile vs. lowest quintile)

TABLE 2 | Subgroup analyses of the association between ALA and risk of HF.

\begin{tabular}{|c|c|c|c|c|c|}
\hline & $\begin{array}{l}\text { Studies } \\
\text { (N) }\end{array}$ & \multicolumn{2}{|c|}{ Quintile 5 vs. Quintile 1} & \multicolumn{2}{|c|}{ Per 1 SD increment } \\
\hline$<65$ & 4 & $0.94[0.81,1.10]$ & $0.85 / 0 \%$ & $0.99[0.93,1.04]$ & $0.85 / 0 \%$ \\
\hline$\geq 65$ & 3 & $0.97[0.83,1.13]$ & & $0.98[0.93,1.03]$ & \\
\hline \multicolumn{6}{|l|}{ Sex } \\
\hline \multicolumn{6}{|c|}{ Follow-up duration (years) } \\
\hline$<10$ years & 3 & $0.90[0.74,1.09]$ & $0.48 / 0 \%$ & $0.96[0.90,1.03]$ & $0.48 / 0 \%$ \\
\hline$\geq 10$ years & 4 & $0.98[0.86,1.10]$ & & $0.99[0.95,1.04]$ & \\
\hline \multicolumn{6}{|c|}{ Measure of exposure } \\
\hline Dietary & 3 & $0.92[0.81,1.04]$ & $0.51 / 0 \%$ & $0.98[0.94,1.02]$ & $0.68 / 0 \%$ \\
\hline
\end{tabular}

${ }^{\star}$ For heterogeneity among subgroups. ALA, $\alpha$-linolenic acid; Cl, confidence intervals; HF, Heart failure; HR, hazard ratios; SD, standard deviation. 
or continuous (per 1-SD) increment of ALA. Furthermore, no heterogeneity was observed among the included studies $\left(\mathrm{I}^{2}=0 \%\right)$ and pooled results were also consistent among comprehensive subgroup analyses. However, some limitations of the current study should be discussed. First, most of the included studies were from the United States, and only one study was from Sweden. The association between ALA and incident HF was still unclear in other populations, especially those with different dietary patterns. Second, we performed this meta-analysis based on the study level. No individual participant data were available, so residual bias cannot be totally avoided. Third, the cardio-protective effects of ALA may be modified by different intakes of LC n-3 PUFAs (37). However, only two studies adjusted the level of DHA and EPA in the analysis. Finally, only one measurement of ALA at baseline was detected in most of the included studies, and the change of ALA overtime was not accounted for. However, the Physicians' Health Study showed that the use of single baseline level ALA, or mean level of between baseline and long-term follow-up measures (up to 15 years), yielded similar associations (both null) on their relationship with HF (38).

\section{CONCLUSIONS}

We did not find any association between ALA (measured either from dietary questionnaires or with a circulating biomarker) and the risk of incident HF. These results suggest that plant origin ALA cannot be regarded as a substitute for LC n-3 fatty acids in the viewpoint for the prevention of HF. Further studies with other populations (e.g., Asians, Africans) are needed to determine whether a high intake of ALA can prevent HF.

\section{REFERENCES}

1. Ziaeian B, Fonarow GC. Epidemiology and aetiology of heart failure. Nat Rev Cardiol. (2016) 13:368-78. doi: 10.1038/nrcardio. 2016.25

2. Mai L, Wen W, Qiu M, Liu X, Sun L, Zheng H, et al. Association between prediabetes and adverse outcomes in heart failure. Diabetes Obes Metab. (2021) 23:2476-83. doi: 10.1111/dom.14490

3. Wu J, Zheng $\mathrm{H}$, Liu X, Chen $\mathrm{P}$, Zhang $\mathrm{Y}$, Luo J, et al. Prognostic value of secreted frizzled-related protein 5 in heart failure patients with and without type 2 diabetes mellitus. Circ Heart Fail. (2020) 13:e7054. doi: 10.1161/CIRCHEARTFAILURE.120.007054

4. Yang S, Chen H, Tan K, Cai F, Du Y, Lv W, et al. Secreted frizzledrelated protein 2 and extracellular volume fraction in patients with heart failure. Oxid Med Cell Longev. (2020) 2020:2563508. doi: 10.1155/2020/ 2563508

5. Jiang W, Whellan DJ, Adams KF, Babyak MA, Boyle SH, Wilson JL, et al. Long-chain omega-3 fatty acid supplements in depressed heart failure patients: results of the OCEAN trial. JACC Heart Fail. (2018) 6:83343. doi: 10.1016/j.jchf.2018.03.011

6. Heydari B, Abdullah S, Shah R, Francis SA, Feng JH, McConnell J, et al. Omega-3 fatty acids effect on post-myocardial infarction ST2 levels for heart failure and myocardial fibrosis. J Am Coll Cardiol. (2018) 72:9535. doi: 10.1016/j.jacc.2018.06.018

\section{DATA AVAILABILITY STATEMENT}

The original contributions presented in the study are included in the article/Supplementary Material, further inquiries can be directed to the corresponding author/s.

\section{AUTHOR CONTRIBUTIONS}

JWu, MQ, LS, D-lL, JWe, and YH: research idea and study design. JWu, MQ, and LS: data acquisition. JWu and MQ: data analysis and interpretation. JWu and LS: statistical analysis. JWu and YH: supervision and mentorship. All authors contributed important intellectual content during manuscript drafting or revision and accept accountability for the overall work by ensuring that questions pertaining to the accuracy or integrity of any portion of the work are appropriately investigated and resolved.

\section{FUNDING}

This project was supported by the Guangdong Basic and Applied Basic Research Fund (Key Project of Guangdong-Foshan Joint Fund) (2019B1515120044), Science and Technology Innovation Project from Foshan, Guangdong (FS0AA-KJ218-1301-0006), and the Clinical Research Startup Program of Shunde Hospital, Southern Medical University (CRSP2019001). The funders had no role in study design, data collection, data analysis, data interpretation, or writing of the report.

\section{SUPPLEMENTARY MATERIAL}

The Supplementary Material for this article can be found online at: https://www.frontiersin.org/articles/10.3389/fcvm. 2021.788452/full\#supplementary-material

7. Djousse L, Akinkuolie AO, Wu JH, Ding EL, Gaziano JM. Fish consumption, omega-3 fatty acids and risk of heart failure: a meta-analysis. Clin Nutr. (2012) 31:846-53. doi: 10.1016/j.clnu.2012.05.010

8. Sakamoto A, Saotome M, Iguchi K, Maekawa Y. Marine-derived omega-3 polyunsaturated fatty acids and heart failure: current understanding for basic to clinical relevance. Int J Mol Sci. (2019) 20:4025. doi: 10.3390/ijms20164025

9. Prasad P, Anjali P, Sreedhar RV. Plant-based stearidonic acid as sustainable source of omega-3 fatty acid with functional outcomes on human health. Crit Rev Food Sci Nutr. (2021) 61:1725-37. doi: 10.1080/10408398.2020.1765137

10. Lázaro I, Rueda F, Cediel G, Ortega E, García-García C, Sala-Vila A, et al. Circulating omega- 3 fatty acids and incident adverse events in patients with acute myocardial infarction. J Am Coll Cardiol. (2020) 76:208997. doi: 10.1016/j.jacc.2020.08.073

11. Naghshi S, Aune D, Beyene J, Mobarak S, Asadi M, Sadeghi O. Dietary intake and biomarkers of alpha linolenic acid and risk of all cause, cardiovascular, and cancer mortality: systematic review and dose-response meta-analysis of cohort studies. BMJ. (2021) 375:n2213. doi: 10.1136/bmj.n2213

12. Wilk JB, Tsai MY, Hanson NQ, Gaziano JM, Djousse L. Plasma and dietary omega-3 fatty acids, fish intake, and heart failure risk in the Physicians' Health Study. Am J Clin Nutr. (2012) 96:882-8. doi: 10.3945/ajcn.112.042671

13. Lemaitre RN, Sitlani C, Song X, King IB, McKnight B, Spiegelman D, et al. Circulating and dietary $\alpha$-linolenic acid and incidence of congestive heart failure in older adults: the Cardiovascular Health Study. Am J Clin Nutr. (2012) 96:269-74. doi: 10.3945/ajcn.112.037960 
14. Belin RJ, Greenland P, Martin L, Oberman A, Tinker L, Robinson J, et al. Fish intake and the risk of incident heart failure: the Women's Health Initiative. Circ Heart Fail. (2011) 4:404-13. doi: 10.1161/CIRCHEARTFAILURE.110.960450

15. Yamagishi K, Nettleton JA, Folsom AR. Plasma fatty acid composition and incident heart failure in middle-aged adults: the Atherosclerosis Risk in Communities (ARIC) study. Am Heart J. (2008) 156:96574. doi: 10.1016/j.ahj.2008.06.017

16. Stroup DF, Berlin JA, Morton SC, Olkin I, Williamson GD, Rennie D, et al. Meta-analysis of observational studies in epidemiology: a proposal for reporting. Meta-analysis Of Observational Studies in Epidemiology (MOOSE) group. JAMA. (2000) 283:2008-12. doi: 10.1001/jama.283.15.2008

17. Wells GA, Shea B, O'Connell D, Peterson J, Welch V, Losos M, Tugwell P. The Newcastle-Ottawa Scale (NOS) for assessing the quality of nonrandomised studies in meta-analyses. Available online at: http://www.ohri.ca/programs/ clinical_epidemiology/oxford.asp (accessed July 15, 2019).

18. Cai X, Zhang Y, Li M, Wu JH, Mai L, Li J, et al. Association between prediabetes and risk of all cause mortality and cardiovascular disease: updated meta-analysis. BMJ. (2020) 370:m2297. doi: 10.1136/bmj.m2297

19. Cai X, Zheng S, Liu Y, Zhang Y, Lu J, Huang Y. Nonalcoholic fatty liver disease is associated with increased risk of atrial fibrillation. Liver Int. (2020) 40:1594-600. doi: 10.1111/liv.14461

20. Li W, Huang A, Zhu H, Liu X, Huang X, Huang Y, et al. Gut microbiotaderived trimethylamine $\mathrm{N}$-oxide is associated with poor prognosis in patients with heart failure. Med J Aust. (2020) 213:374-9. doi: 10.5694/mja2.50781

21. Yang Y, Li W, Zhu H, Pan XF, Hu Y, Arnott C, et al. Prognosis of unrecognised myocardial infarction determined by electrocardiography or cardiac magnetic resonance imaging: systematic review and meta-analysis. BMJ. (2020) 369:m1184. doi: 10.1136/bmj.m1184

22. Levitan EB, Wolk A, Håkansson N, Mittleman MA. $\alpha$-Linolenic acid, linoleic acid and heart failure in women. Br J Nutr. (2012) 108:13006. doi: 10.1017/S0007114511006726

23. Djousse L, Biggs ML, Matthan NR, Ix JH, Fitzpatrick AL, King I, et al. Serum individual nonesterified fatty acids and risk of heart failure in older adults. Cardiology. (2021) 146:351-8. doi: 10.1159/000513917

24. Asselin CY, Lam A, Cheung D, Eekhoudt CR, Zhu A, Mittal I, et al. The cardioprotective role of flaxseed in the prevention of doxorubicinand trastuzumab-mediated cardiotoxicity in C57BL/6 mice. J NUTR. (2020) 150:2353-63. doi: 10.1093/jn/nxaa144

25. Yu X, Cui L, Zhang Z, Zhao Q, Li S. $\alpha$-Linolenic acid attenuates doxorubicin-induced cardiotoxicity in rats through suppression of oxidative stress and apoptosis. Acta Biochim Biophys Sin. (2013) 45:817-26. doi: 10.1093/abbs/gmt082

26. Leung KS, Galano JM, Oger C, Durand T, Lee JC. Enrichment of alphalinolenic acid in rodent diet reduced oxidative stress and inflammation during myocardial infarction. Free Radic Biol Med. (2021) 162:5364. doi: 10.1016/j.freeradbiomed.2020.11.025

27. Parikh M, Raj P, Austria JA Yu L, Garg B, Netticadan T, Pierce GN. Dietary flaxseed protects against ventricular arrhythmias and left ventricular dilation after a myocardial infarction. J Nutr Biochem. (2019) 71:6371. doi: 10.1016/j.jnutbio.2019.06.004

28. Russell JS, Griffith TA, Naghipour S, Vider J, Du Toit EF, Patel HH, et al. Dietary $\alpha$-linolenic acid counters cardioprotective dysfunction in diabetic mice: unconventional PUFA protection. Nutrients. (2020) 12:2679. doi: 10.3390/nu12092679

29. Barbeau PA, Holloway TM, Whitfield J, Baechler BL, Quadrilatero J, van Loon $\mathrm{L}$, et al. $\alpha$-Linolenic acid and exercise training independently, and additively, decrease blood pressure and prevent diastolic dysfunction in obese Zucker rats. J Physiol. (2017) 595:4351-64. doi: 10.1113/JP274036

30. Saeedi SS, Bonetti NR, Pugin B, Constancias F, Pasterk L, Gobbato $\mathrm{S}$, et al. Lifelong dietary omega-3 fatty acid suppresses thrombotic potential through gut microbiota alteration in aged mice. iScience. (2021) 24:102897. doi: 10.1016/j.isci.2021.102897

31. Duda MK, O'Shea KM, Tintinu A, Xu W, Khairallah RJ, Barrows BR, et al. Fish oil, but not flaxseed oil, decreases inflammation and prevents pressure overload-induced cardiac dysfunction. Cardiovasc Res. (2009) 81:319-27. doi: 10.1093/cvr/cvn310

32. Yue H, Qiu B, Jia M, Liu W, Guo XF, Li N, et al. Effects of $\alpha$-linolenic acid intake on blood lipid profiles: a systematic review and meta-analysis of randomized controlled trials. Crit Rev Food Sci Nutr. (2020) 61:2894910. doi: 10.1080/10408398.2020.1790496

33. Joris PJ, Draijer R, Fuchs D, Mensink RP. Effect of $\alpha$-linolenic acid on vascular function and metabolic risk markers during the fasting and postprandial phase: a randomized placebo-controlled trial in untreated (pre-)hypertensive individuals. Clin Nutr. (2020) 39:2413-9. doi: 10.1016/j.clnu.2019.11.032

34. Farley G, Riggs DW, Bhatnagar A, Hellmann J. Omega-3 polyunsaturated fatty acids modify the inverse association between systemic inflammation and cardiovascular fitness. Clin Nutr. (2021) 40:4097-105. doi: 10.1016/j.clnu.2021.02.006

35. Bersch-Ferreira AC, Hall WL, Santos R, Torreglosa CR, Sampaio G, Tereza DSJ, et al. The effect of the a regional cardioprotective nutritional program on inflammatory biomarkers and metabolic risk factors in secondary prevention for cardiovascular disease, a randomised trial. Clin Nutr. (2021) 40:382835. doi: 10.1016/j.clnu.2021.04.035

36. Block RC, Liu L, Herrington DM, Huang S, Tsai MY, O'Connell TD, et al. Predicting Risk for Incident Heart Failure With Omega-3 Fatty Acids: From MESA. JACC Heart Fail. (2019) 7:651-61. doi: 10.1016/j.jchf.2019. 03.008

37. Bork CS, Lundbye-Christensen S, Venø SK, Lasota AN, Schmidt EB, Overvad K. Plant n-3 PUFA intake may lower the risk of atherosclerotic cardiovascular disease only among subjects with a low intake of marine n-3 PUFAs. Eur J Nutr. (2021). doi: 10.1007/s00394-021-02581-5. [Epub ahead of print].

38. Djousse L, Petrone AB, Weir NL, Hanson NQ, Glynn RJ, Tsai MY, et al. Repeated versus single measurement of plasma omega- 3 fatty acids and risk of heart failure. Eur J Nutr. (2014) 53:1403-8. doi: 10.1007/s00394-013-0642-3

Conflict of Interest: The authors declare that the research was conducted in the absence of any commercial or financial relationships that could be construed as a potential conflict of interest.

Publisher's Note: All claims expressed in this article are solely those of the authors and do not necessarily represent those of their affiliated organizations, or those of the publisher, the editors and the reviewers. Any product that may be evaluated in this article, or claim that may be made by its manufacturer, is not guaranteed or endorsed by the publisher.

Copyright (C) 2022 Wu, Qiu, Sun, Wen, Liang, Zheng and Huang. This is an openaccess article distributed under the terms of the Creative Commons Attribution License (CC BY). The use, distribution or reproduction in other forums is permitted provided the original author(s) and the copyright owner(s) are credited and that the original publication in this journal is cited, in accordance with accepted academic practice. No use, distribution or reproduction is permitted which does not comply with these terms. 